\title{
Inverse Schwartz-Christoffel panel method
}

\author{
E. Morishita \\ Department of Mechanical Engineering, \\ School of Science and Engineering, Meisei University, Japan
}

\begin{abstract}
A two-dimensional panel method is proposed based on the Schwartz-Christoffel transformation from a unit circle to a polygonal aerofoil. Although numerical integration of the panel length is necessary, this method gives analytical solution for an aerofoil approximated as a polygon. It is also found that this method solves an extremely thin aerofoil problem properly. This is because the solution is simply an analytical transformation of the potential flow around a circular cylinder. In this paper, an inverse design method of a two-dimensional aerofoil is proposed based on the Schwartz-Christoffel transformation. The velocity distribution in the physical plane is given. Calculations are conducted backward from the solutions of symmetric Joukowski and NACA0012 aerofoils together with non-symmetric NACA4412 where simultaneous equations are solved to get the aerofoil. A non-linear inverse method is finally proposed. Triangular and NACA aerofoils are tested to show the concept.
\end{abstract}

Keywords: aerofoil, inverse design, conformal mapping.

\section{Introduction}

The Schwartz-Christoffel panel method has been proposed. A unit circle is transformed to a polygon. A potential flow around a circular cylinder becomes that around an aerofoil approximated as a polygon. It is important to note that a flow around an aerofoil is an analytical solution although this method requires numerical integration of the panel length.

During the course of the development of the technique, it is found that an analytical formula of the velocity at a collocation point of a panel inversely becomes a linear equation of the panel inclinations when the velocity distribution in the physical $\zeta$-plane with nodes and the angle of attack in the original $z$-plane 
are given. This method is first explained and then a possible full inverse method is proposed based on velocity and/or pressure at given coordinates.

\section{Schwartz-Christoffel panel method}

Points $z_{j}=e^{i \theta_{j}}\left(1 \leq j \leq N, \theta_{1}=0<\theta_{2}<\ldots<\theta_{N}<2 \pi\right)$ on a unit circle in the $z$-plane can be transformed to a $N$ polygonal aerofoil in the $\zeta$-plane based on the Schwartz-Christoffel transformation given by [1-3]

$$
\frac{d \zeta}{d z}=A \cdot \prod_{j=1}^{N}\left(1-\frac{z_{j}}{z}\right)^{\mu_{j}},
$$

where $A \equiv K e^{i \kappa}$ is a complex constant with real constants $K$ and $\kappa$.

Figure 1 shows the result of a Joukowski aerofoil with $N=40$ where $z_{0}$ is the centre of a base circle in the original $z$-plane and $\alpha$ is an angle of attack. Agreement with the theory is quite satisfactory, cf. $[4,5]$.

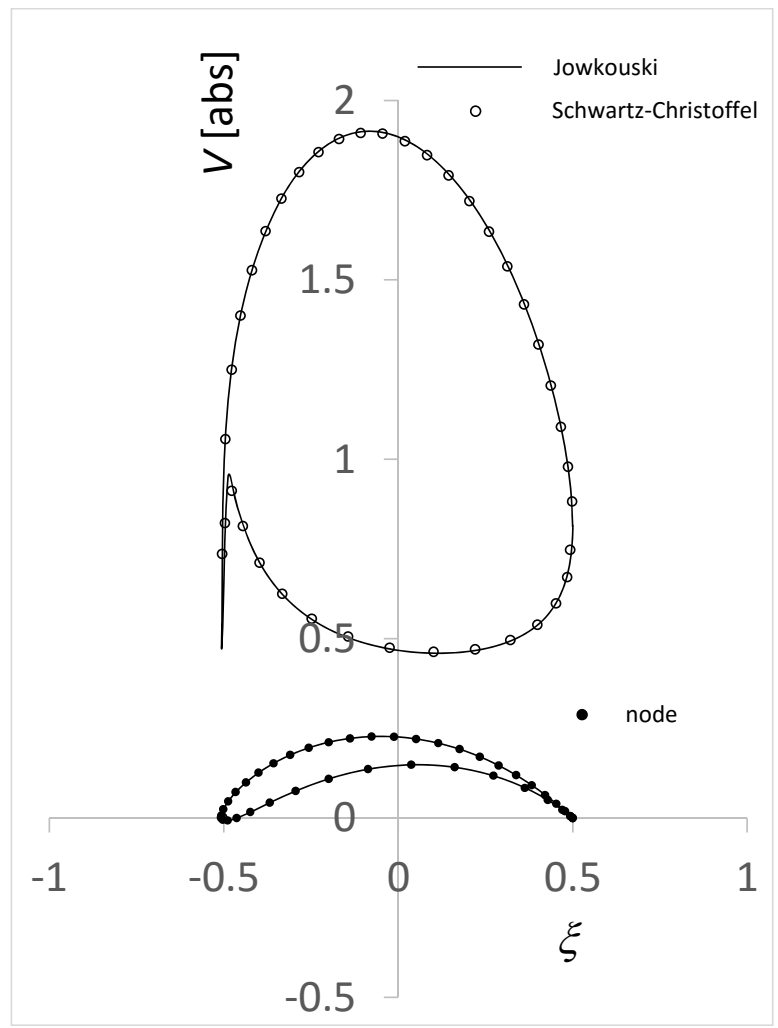

Figure 1: Surface velocity $|V|$ of a Joukowski aerofoil at $\alpha=0^{\circ}$, $z_{0}=(-0.02,0.1)$. 


\section{Backward calculation from known solutions}

\subsection{Symmetric aerofoil}

For simplicity, a backward calculation from known solutions is conducted. A symmetric velocity distribution $V_{i}$ on the node $i$ of the physical $\zeta$-plane is given where the values of $\theta_{i}(1 \leq i \leq N)$ in the original $z$-plane are incidentally known as shown in fig. 2. This is not the case for the inverse process. This is actually a Joukowski aerofoil which shape has to be determined.

From the Schwartz-Christoffel transformation [2]

$$
\frac{\sin \left(\theta_{i \text { mid }}-\alpha_{z}\right)+\sin \alpha_{z}}{2 \prod_{j=1}^{N}\left|\sin \frac{\theta_{j}-\theta_{i \text { mid }}}{2}\right|^{\mu_{j}}}=\frac{V_{i}}{V_{\infty}}=(1 \leq i \leq N),
$$

where $V_{i}$ is the panel velocity and $V_{\infty}$ is the uniform velocity, respectively, in the $\zeta$-plane, $\alpha_{z}$ is the angle of attack in the $z$-plane, $\mu_{j}(1 \leq j \leq N)$ is the aerofoil panel turning angle ratio relative to $\pi$, and

$$
\theta_{i \text { mid }} \equiv \frac{\theta_{i}+\theta_{i+1}}{2}(1 \leq i \leq N) \text {. }
$$

Note that $\theta_{i \text { mid }}$ does not necessarily represent the panel midpoint.

Logarithmic form of eqn (2) becomes

$$
\begin{aligned}
& \ln \left|\sin \frac{\theta_{1}-\theta_{i \text { mid }}}{2}\right| \cdot \mu_{1}+\ln \left|\sin \frac{\theta_{2}-\theta_{i \text { mid }}}{2}\right| \cdot \mu_{2}+\ldots \ln \left|\sin \frac{\theta_{N}-\theta_{i \text { mid }}}{2}\right| \cdot \mu_{N} \\
& =\ln \frac{\sin \left(\theta_{i \text { mid }}-\alpha_{z}\right)+\sin \alpha_{z}}{2 \cdot\left(\frac{V_{i}}{V_{\infty}}\right)}
\end{aligned}
$$

Because of symmetry, eqn (4) becomes for an even number $N$

$$
\begin{aligned}
& \left.\ln \left|\sin \frac{\theta_{1}-\theta_{i \text { mid }}}{2}\right| \cdot \mu_{1}+|\ln | \sin \frac{\theta_{2}-\theta_{i \text { mid }}}{2}|+\ln | \sin \frac{\theta_{N}-\theta_{i \text { mid }}}{2} \mid\right] \cdot \mu_{2} \\
& \left.+|\ln | \sin \frac{\theta_{3}-\theta_{i \text { mid }}}{2}|+\ln | \sin \frac{\theta_{N-1}-\theta_{i \text { mid }}}{2} \mid\right] \cdot \mu_{3}+\ldots \\
& \left.+|\ln | \sin \frac{\frac{\theta_{N}}{2}-\theta_{i \text { mid }} \mid}{2}|+\ln | \sin \frac{\frac{\theta_{N}}{2}-\theta_{i \text { mid }}}{2} \mid\right] \cdot \mu_{\frac{N}{2}}+\ln \left|\sin \frac{\frac{\theta_{N}}{2}-\theta_{i \text { mid }} \mid}{2}\right| \cdot \mu_{\frac{N}{2}+1}, \\
& =\ln \frac{\sin \left(\theta_{i \text { mid }}-\alpha_{z}\right)+\sin \alpha_{z}}{2\left(\frac{\bar{V}_{i}}{V_{\infty}}\right)} \quad\left(1 \leq i \leq \frac{N}{2}\right)
\end{aligned}
$$




$$
\begin{aligned}
& \mu_{1}+\mu_{2}+\mu_{3} \ldots+\mu_{\frac{N}{2}}+\mu_{\frac{N}{2}+1}+\mu_{\frac{N}{2}+2}+\ldots+\mu_{N-1}+\mu_{N} \\
& =\mu_{1}+2\left(\mu_{2}+\mu_{3}+\ldots+\mu_{\frac{N}{2}}\right)+\mu_{\frac{N}{2}+1}=2 .
\end{aligned}
$$

The unknown $\mu_{i}\left(1 \leq i \leq \frac{N}{2}+1\right)$ can be determined by solving the simultaneous equations, eqns (5) and (6) obtained above, and the rest of $\mu_{i}\left(\frac{N}{2}+2 \leq i \leq N\right)$ are also obtained from the symmetry.

For the symmetric aerofoil, the chord inclination is zero, and

$$
\kappa=0 \text {. }
$$

The aerofoil coordinates $\zeta_{i}=\left(\xi_{i}, \eta_{i}\right)$ become [2]

$$
\begin{aligned}
& \frac{\xi_{i}}{K}=\frac{\xi_{1}}{K}+\sum_{j=1}^{i-1} \frac{l_{j}}{K} \cdot \cos \delta_{j}, \\
& \frac{\eta_{i}}{K}=\frac{\eta_{1}}{K}+\sum_{j=1}^{i-1} \frac{l_{j}}{K} \cdot \sin \delta_{j},
\end{aligned}
$$

where $l_{j}(1 \leq j \leq N)$ is the panel length, and $\delta_{j}(1 \leq j \leq N)$ is the panel inclination angle, respectively. $\left(\xi_{1} / K, \eta_{1} / K\right)$ is the trailing edge coordinate and it can be chosen arbitrary, and most typically $(1,0)$. The constant $K$ can be determined from the chord length $c$.

Figures 2 and 3 represent Joukowski $\left[\zeta=z+(1 / 4)^{2} / z, z_{0}=(-0.02,0)\right]$ and NACA0012 aerofoils, respectively. Note that these results are obtained from the known answers just to show the backward calculation procedure.

Numerical irregularity in $\mu_{i}$ might appear even when the matrix of a solved symmetric aerofoil is inverted. In this case, the velocity from known $\mu_{i}$ is recalculated intentionally, and then the matrix is inverted again to get regular solutions of $\mu_{i}$ to show the process. Slight numerical error may cause the irregularity.

\subsection{Non-symmetric aerofoil}

In eqns (4), the angles $\theta_{i}(1 \leq i \leq N)$ and $\alpha_{z}$ are given in the original $z$-plane, and $V_{i} / V_{\infty}$ is known in the physical $\zeta$-plane. Care must be taken that although the node number $i$ is given, $\xi_{i}$ is not given.

Equations (4) are solved, and $\mu_{i}(1 \leq i \leq N)$ are obtained; see fig. 4(c). Equations (8) and (9) give the aerofoil shape as in fig. 4(d). Figure 4(d) can be rotated and contracted to fig. $4(\mathrm{e})$ with $A=K e^{i \kappa}(1 / K \approx 3.656, \kappa \approx-0.0726)$. The velocity and the pressure distribution are finally obtained in fig. $4(\mathrm{f})$ and fig. $4(\mathrm{~g})$. 


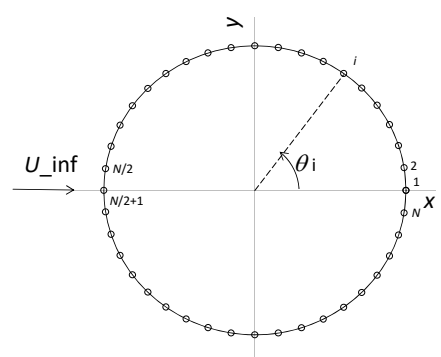

(a) $\theta_{i}(1 \leq i \leq N) \alpha_{z}=0^{\circ}$

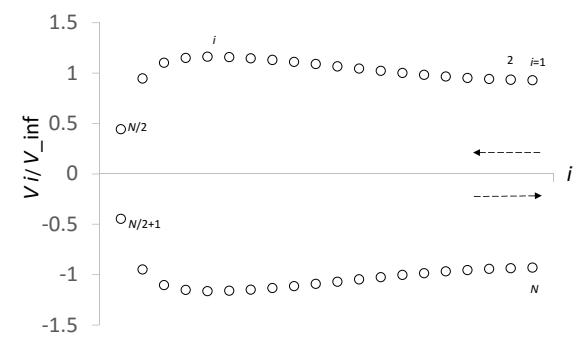

(b) $\frac{V_{i}}{V_{\infty}}(1 \leq i \leq N)$ given: node $i$ of the $\zeta$-plane

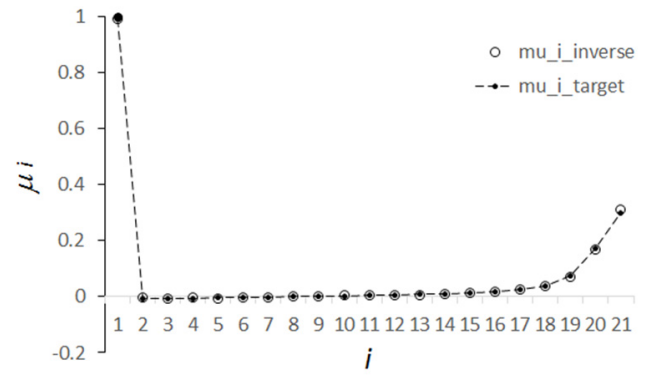

(c) inverse $\mu_{i} \quad\left(1 \leq i \leq \frac{N}{2}+1\right)$

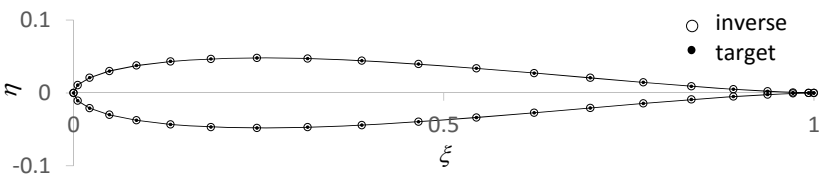

$(d)$ inverse $\zeta_{i}=\left(\xi_{i}, \eta_{i}\right) \quad(1 \leq i \leq N)$

Figure 2: Backward calculation of a symmetric Joukowski aerofoil $\frac{N}{2}+1=21$. 


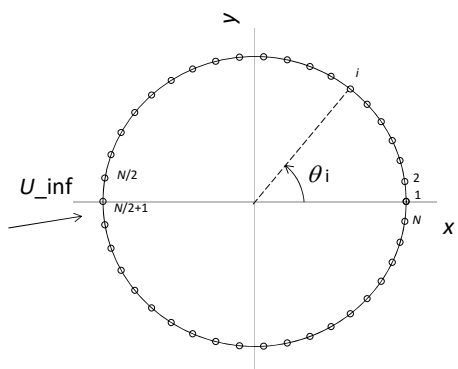

(a) $\theta_{i}(1 \leq i \leq N), \alpha_{z}=4^{\circ}$

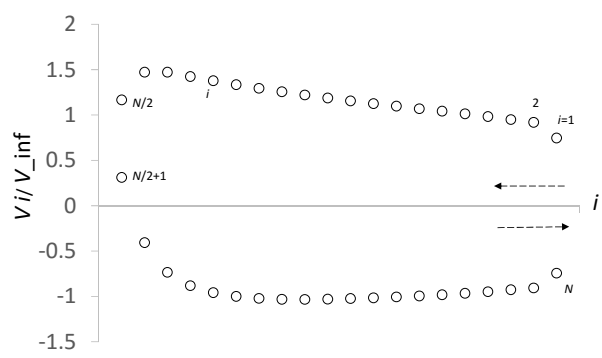

(b) $\frac{V_{i}}{V_{\infty}}(1 \leq i \leq N)$ given: node $i$ of the $\zeta$-plane.

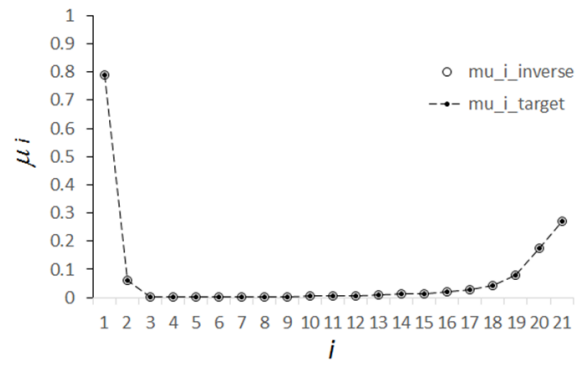

(c) inverse $\mu_{i} \quad\left(1 \leq i \leq \frac{N}{2}+1\right)$

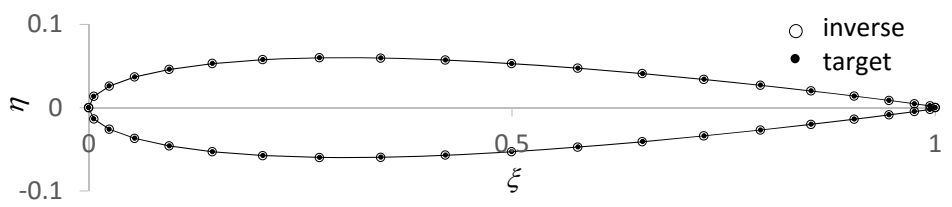

$(\mathrm{d})$ inverse $\zeta_{i}=\left(\xi_{i}, \eta_{i}\right) \quad(1 \leq i \leq N)$

Figure 3: Backward calculation of a symmetric NACA0012 aerofoil $\frac{N}{2}+1=21$. 


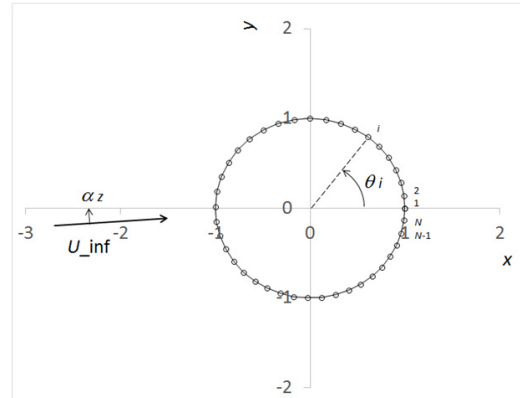

(a) $\alpha_{z}, \theta_{i}(1 \leq i \leq N)$ given: $z$ plane

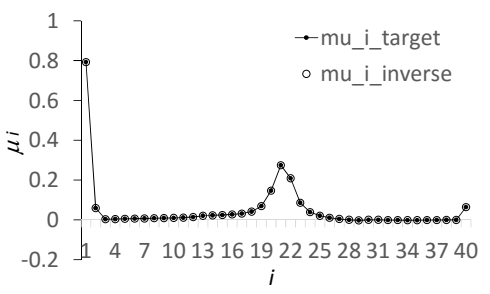

(c) inverse $\mu_{i}$

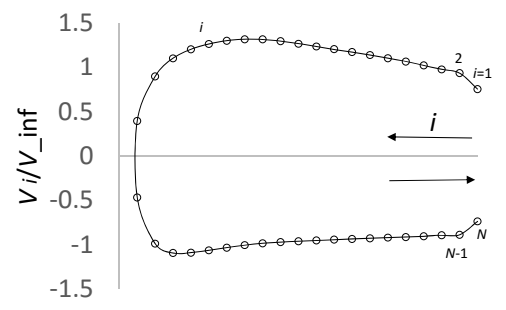

(b) $V_{i} / V_{\infty}$ given: node $i$ of the $\zeta$ plane

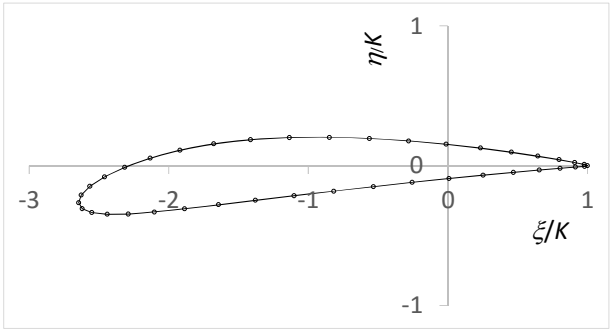

(d) inverse $\frac{\zeta_{i}}{K}$

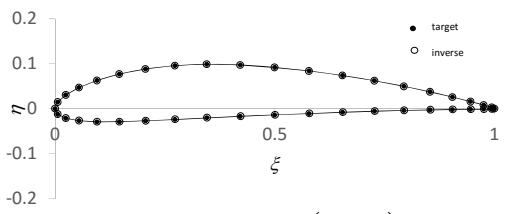

(e) inverse $\zeta_{i}=\left(\xi_{i}, \eta_{i}\right)$

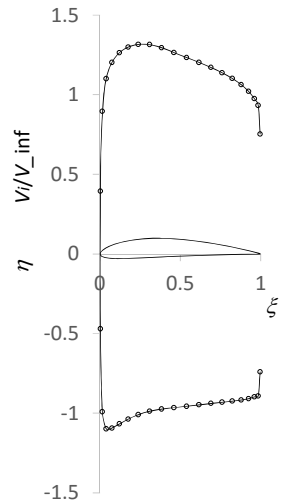

(f) surface velocity

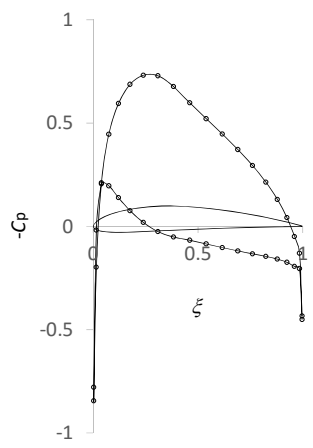

(g) pressure distribution

Figure 4: Backward calculation of a non-symmetric NACA4412 aerofoil $\alpha=0^{\circ} \quad\left(\alpha_{z}=-\kappa \approx 4.2^{\circ}\right)$. 


\section{Inverse method}

\subsection{Inverse method for a triangular aerofoil}

The velocities $V_{[i]}(1 \leq i \leq 3)$ and the corresponding coordinates $\xi_{[i]}(1 \leq[i] \leq 3)$ are given; see black symbols in fig. 5. Six conditions are known and these might determine the triangle. The trailing edge locates at $\zeta_{1}=1$, and the leading edge $\zeta_{3}$ at the origin. The following equations must be satisfied in this case:

$$
\begin{aligned}
& \mu_{1}+\mu_{2}+\mu_{3}=2 \text {, } \\
& \mu_{1} \cdot \cos \theta_{1}+\mu_{2} \cdot \cos \theta_{2}+\mu_{3} \cdot \cos \theta_{3}=0, \\
& \mu_{1} \cdot \sin \theta_{1}+\mu_{2} \cdot \sin \theta_{2}+\mu_{3} \cdot \sin \theta_{3}=0, \\
& \frac{\sin \left(\theta_{\xi[i]}-\alpha_{z}\right)+\sin \alpha_{z}}{2 \prod_{j=1}^{3}\left|\sin \frac{\theta_{j}-\theta_{\xi_{[i]}}}{2}\right|^{\mu_{j}}}=\frac{V_{[i]}}{V_{\infty}} \quad(1 \leq i \leq 3), \\
& \left.\operatorname{Re}\left[\zeta_{1}+l \mid \theta_{1}, \theta_{\xi_{[1]}}\right\rfloor \cdot e^{i \delta_{1}}\right\rfloor=\xi_{[1]}, \\
& \left.\operatorname{Re}\left\lfloor\zeta_{1}+l_{1} \cdot e^{i \delta_{1}}+l \mid \theta_{2}, \theta_{\xi[2]}\right\rfloor \cdot e^{i \delta_{2}}\right\rfloor=\xi_{[2]}, \\
& \left.\operatorname{Re}\left\lfloor\zeta_{3}+l \mid \theta_{3}, \theta_{\xi_{[3]}}\right\rfloor \cdot e^{i \delta_{3}}\right\rfloor=\xi_{[3]},
\end{aligned}
$$

where

$$
l\left[\theta_{i}, \theta\right] \equiv K \cdot 2^{2} \cdot \int_{\theta_{i}}^{\theta} \prod_{j=1}^{3}\left|\sin \frac{\theta_{j}-\theta}{2}\right|^{\mu_{j}} \cdot d \theta .
$$

The eight unknowns $\theta_{2}, \theta_{3}, \theta_{\xi[1]}, \theta_{\xi[2]}, \theta_{\xi[3]}, \mu_{1}, \mu_{2}$, and $\alpha_{z}$ can be varied to satisfy the above eight eqns (10)-(17), simultaneously. The initial values can be chosen as $K=1$ and $\kappa=0$. The constants $K$ and $\kappa$ are varied properly to adjust the chord length and its inclination, see fig. 5(b). Spreadsheet solver function is very useful for this problem. The angle $\theta_{1}$ can be set $\theta_{1}=0$, but $\mu_{3}$ is not an independent variable due to eqn (10). The angle of attack $\alpha$ is given by $\alpha=\alpha_{z}+\kappa$ [2].

\subsection{Inverse method by panel average velocity for a triangular aerofoil}

The panel average velocities $\bar{V}_{i}(1 \leq i \leq 3)$ and the coordinates in fig. 6 become [2]

$$
\begin{gathered}
-2\left(\frac{K}{l_{i}}\right) \cdot\left[\cos \left(\theta_{i+1}-\alpha_{z}\right)-\cos \left(\theta_{i}-\alpha_{z}\right)-\left(\theta_{i+1}-\theta_{i}\right) \cdot \sin \alpha_{z}\right]=\frac{\bar{V}_{i}}{V_{\infty}}, \\
\operatorname{Re}\left[\zeta_{1}\right]=\xi_{1}(=0), \\
\operatorname{Re}\left[\zeta_{2}\right]=\operatorname{Re}\left[\zeta_{1}+l_{1} \cdot e^{i \delta_{1}}\right]=\xi_{2}, \\
\operatorname{Re}\left[\zeta_{3}\right]=\operatorname{Re}\left[\zeta_{1}+l_{1} \cdot e^{i \delta_{1}}+l_{2} \cdot e^{i \delta_{2}}\right]=\xi_{3}\left(=\zeta_{\text {l.e. }}=0\right) .
\end{gathered}
$$




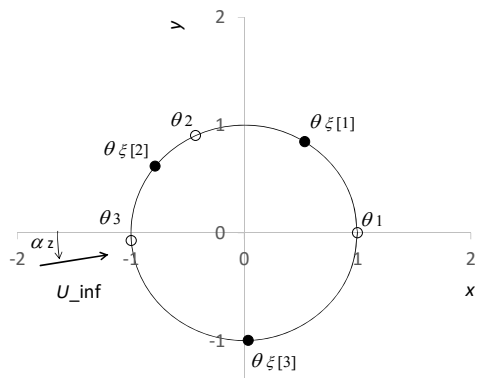

(a) $z$-plane

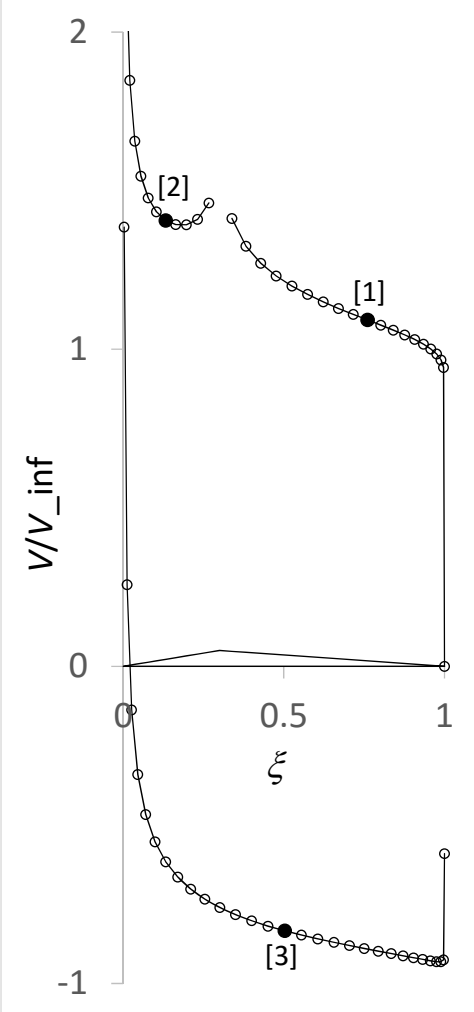

(c) velocity
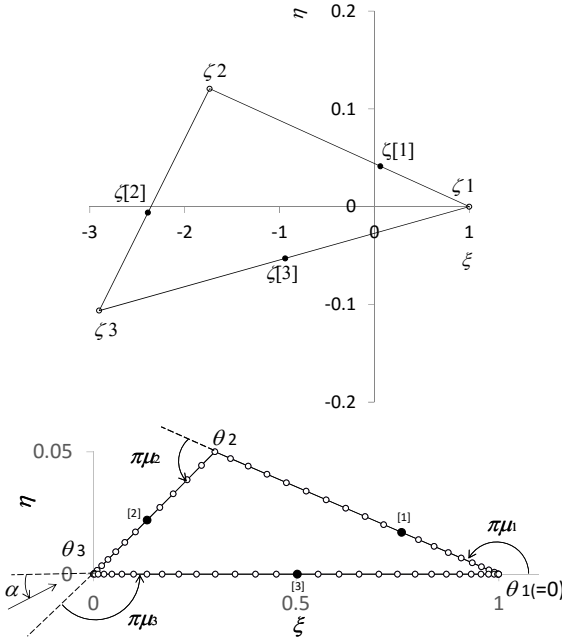

(b) $\zeta$-plane: scaled by $A=K e^{i \kappa}$

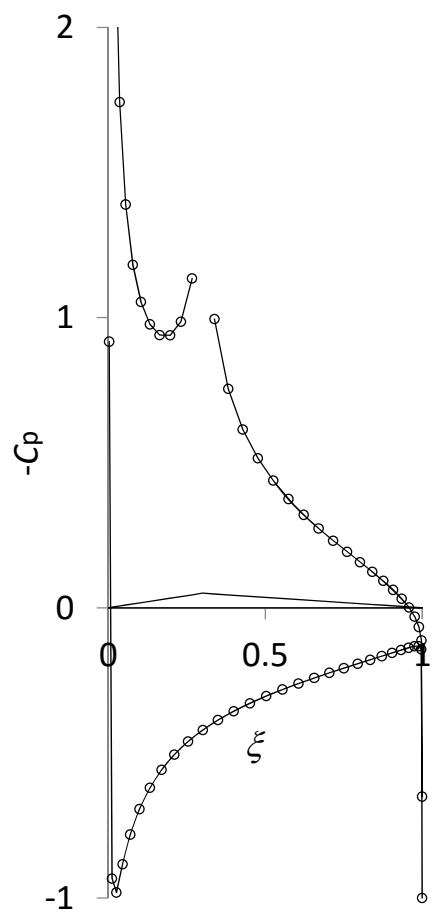

(d) pressure

Figure 5: Triangular aerofoil $\left(\alpha=8^{\circ}, 5 \%\right.$ thickness at $\left.\xi=0.3\right)$. 


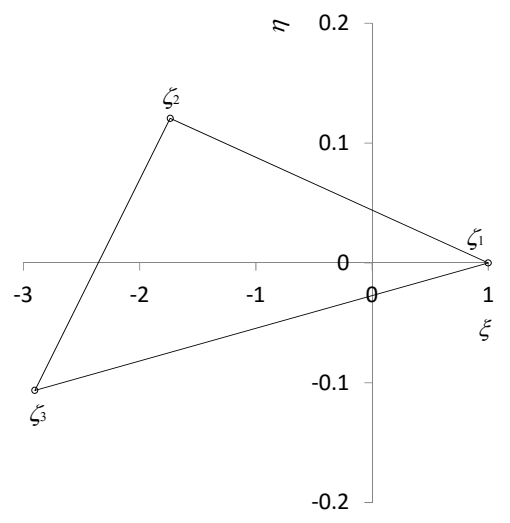

(a) $K=1, \kappa=0$

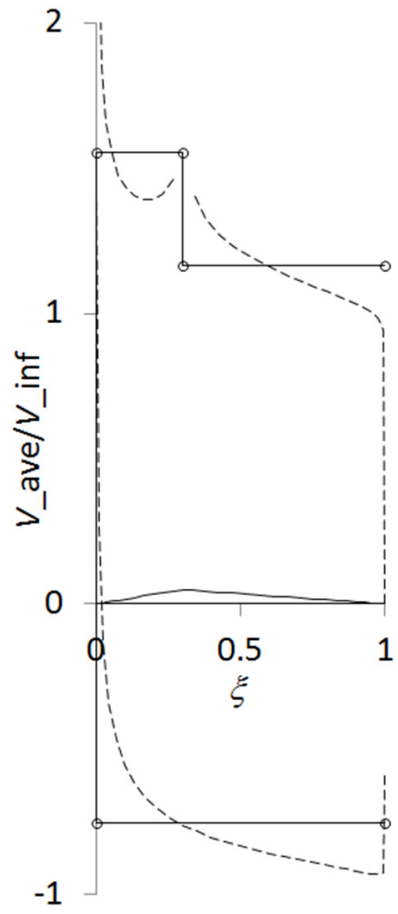

(b) scaled by $A=K e^{i \kappa}$

Figure 6: Panel average velocity of the triangular aerofoil in fig. 5.

For computational simplicity, the average velocity $\bar{V}_{i}$ is given at $\xi=\xi_{i}$, i.e., at the very beginning of the panel $i$, not at the middle of the panel. Equation (19) is a given constant, and therefore five conditions are given altogether, eqn (18) and eqns (20) and (21). The five unknowns $\theta_{2}, \theta_{3}, \mu_{1}, \mu_{2}$, and $\alpha_{z}$ are varied by the solver function again. The constants $K$ and $\kappa$ can be determined from the chord length and its inclination, see fig. 5(b) and fig. 6.

The geometrical requirement to form a closed triangle becomes

$$
\sum_{i=1}^{3} l_{i} \cdot e^{i \delta_{i}}=0 .
$$

Equations (20) and (21) can be replaced by eqn (22).

\subsection{Inverse method by panel average velocity for an aerofoil}

A polygonal aerofoil is a natural extension of a triangular aerofoil. The panel average velocities $\bar{V}_{i}\left(1 \leq i \leq N, \xi_{i} \leq \xi \leq \xi_{i+1}\right)$ and their lateral coordinates $\xi_{i}(1 \leq i \leq N)$ are given in the physical $\zeta$-plane. The trailing edge $\xi_{1}\left(=\xi_{\text {t.e. }}=1\right)$ is 
a given constant, and effective conditions become $2 N-1$. The first angle can be set $\theta_{1}=0$, and one of $\mu_{i}(1 \leq i \leq N)$, for example $\mu_{1}$, is not independent. The $2 N-1$ unknowns $\theta_{2}, \theta_{3}, \ldots, \theta_{N}, \mu_{2}, \mu_{3}, \ldots, \mu_{N}$ and $\alpha_{z}$ must be determined.

The following equation is applied to form a closed polygon:

$$
\sum_{i=1}^{N} l_{i} \cdot e^{i \delta_{i}}=0 .
$$

So another two necessary conditions are given by eqn (23). As to the two unknowns $K$ and $\kappa$, their values can be determined from the chord length and its inclination.

Small deviations from the known answers are given to get the final solution. The concept is tested in this manner. Figure 7(a) shows trial initial conditions. The target aerofoil is shown in fig. 7(b).

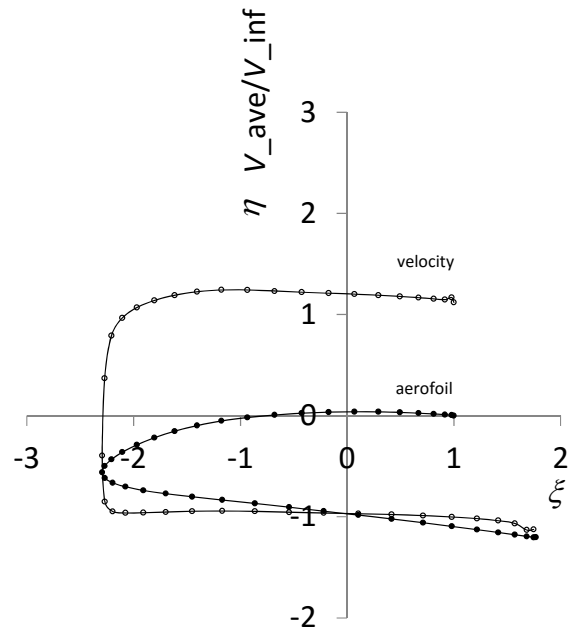

(a) initial configuration $\left[\xi=\xi_{i}=\xi\left(\theta_{i}\right)\right]$

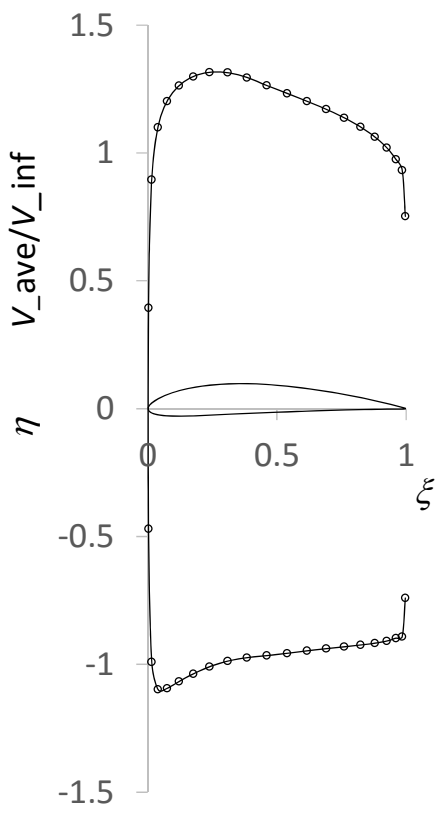

(b) solution $\left\lfloor\xi=\xi_{i \text { mid }}=\xi\left(\theta_{i \text { mid }}\right)\right\rfloor$

Figure 7: NACA4412 aerofoil $\alpha=0^{\circ}$.

Initial conditions:

$$
\begin{aligned}
& \theta_{i}=\frac{2 \pi}{N}(i-1), \mu_{i}=0.9 \times \mu_{i \text { exact }}(2 \leq i \leq N), \\
& \mu_{1}=2-\sum_{i=2}^{N} \mu_{i}, K=1, \kappa=0, \alpha_{z}=0
\end{aligned}
$$




\section{Concluding remarks}

A novel inverse Schwartz-Christoffel panel method is proposed. First the backward calculation is conducted from the known solutions. A basic flow in the original $z$-plane and discrete velocities in the physical plane are given. Simultaneous linear equations of the panel turning angle ratio are derived from the analytical formula of the velocity on a panel collocation point. The panel turning angle ratio is inversely calculated and construct a target aerofoil. Several illustrative examples are given from known solutions. The present inverse method is then introduced where velocity and/or pressure are given at known lateral coordinates. A triangular aerofoil is a test case and this novel inverse method is applied to a NACA aerofoil to show the concept.

\section{References}

[1] Moriya, T., Introduction to Aerodynamics (in Japanese), Baifu-kan: Tokyo, p. 139, 1977.

[2] Morishita, E., Schwartz-Christoffel Panel Method Improvements and Applications, Computational Problems in Science and Engineering, Lecture Notes Electrical Engineering 343, Springer: Berlin, pp. 109-128, 2015.

[3] Saenz, L., The Schwarz-Christoffel panel method: a computational kit, www.maplesoft.com/applications/view.aspx?SID=153963, 2016.

[4] Moran, J., An Introduction to Theoretical and Computational Aerodynamics, John Wiley \& Sons: New York, p. 286, 1984.

[5] Tanaka, S., Murata, S., \& Karata, K, Computation of the Potential Flow through Cascades using the Conformal Mapping and the Singularity Method, JSME International Journal, Series II,34, pp. 423-430, 1991. 